\title{
SYMMETRIC INTEGRO-DIFFERENTIAL-BOUNDARY PROBLEMS
}

BY

\author{
HYMAN J. ZIMMERBERG
}

ABSTRACT. Necessary and sufficient conditions for a linear vector integrodifferential-boundary problem to be symmetric (selfadjoint) are developed, and then applied to obtain canonical forms of such symmetric problems. Moreover, the formulation of the integro-boundary conditions herein yields a simplification of one of the conditions for selfadjointness of a differential-boundary operator previously announced.

1. Introduction. Necessary and sufficient conditions for a class of differential-boundary operators to be selfadjoint, recently given by Krall [3], will be extended to integro-differential-boundary problems, in vector form,

$$
A_{1}(x) y^{\prime}+A_{0}(x) y+H(x)\left[M_{2} y(a)+N_{2} y(b)\right]+K(x) \int_{a}^{j} F(t) y d t=\lambda B(x) y
$$

$$
M y(a)+N y(b)+\int_{a}^{b} F(t) y d t=0
$$

In addition, for the special case $K(x) \equiv 0$ the formulation of the conditions (1.1b) herein allows a simplification of one of the conditions in [3].

A suitable formulation of a problem adjoint to a differential system with integral-boundary conditions has been the subject of a number of papers, among them [1], [2], [3] and [5]. However, the definition of symmetry (se lfadjointness) adopted in this paper is more in accordance with that employed for boundary problems (see, for example, [4, p. 197]) than the requirement used in [3]. Moreover, while the integral term in (1.1a) may be replaced by a boundary term in view of condition (1.1b), nevertheless, there is an extra measure of generality in this setting that is unavailable in [3].

Presented to the Society, October 27, 1973; received by the editors March 27, 1973.

AMS (MOS) subject classifications (1970). Primary 34A30, 34B05, 47G05; Secondary 34A20, 47A05, 47A50, 47D99.

Key words and phrases. Adjoint, boundary value problem, canonical form, differential system, integro-boundary condition, integro-differential-boundary problem, linear, operator, selfadjoint, symmetric. 
The adjoint problem will be devloped and necessary and sufficient conditions for symmetry will be derived in $\$ 2$ for problems wherein the form of the integralboundary condition (1.1b) is an extension of that used in [5]. Canonical forms for classes of equivalent symmetric integro-differential-boundary problems (1.1a), (1.1b) will then be constructed in $\$ 3$.

Matrix and vector notation will be employed throughout. Matrices of various dimensional orders will be denoted by both italic and Greek capital letters, while vectors will be represented by lower-case italic letters. The operations of differentiation and conjugate-transpose for both matrices and vectors will be indicated by ' and *, respectively. Moreover, as is customary, 0 will be used indiscriminately to denote either the number zero, a zero matrix or a zero vector; the $\sigma \times \sigma$ identity matrix will be indicated by $I_{\sigma}$, and $i$ will denote a complex square root of -1 . Finally, when row and column dimensions agree, $[M, N ; P, Q]$ will represent the matrix $\left[\begin{array}{cc}M & N \\ P & Q\end{array}\right]$.

2. Necessary and sufficient conditions. For the problem (1.1a), (1.1b) it will be assumed that the elements of the $n \times n$ matrix $A_{1}(x)$ are of class $C^{\prime}$ on the finite interval $a \leq x \leq b$, the elements of the $n \times n$ matrices $A_{0}(x)$ and $B(x)$, the $m \times n$ matrix $F(x)$, the $n \times m$ matrix $K(x)$, and the $n \times p$ matrix $H(x)$ are continuous on $[a, b], \lambda$ is a scalar parameter, $M_{2}$ and $N_{2}$ are each $p \times n$ constant matrices, and $M$ and $N$ are each $m \times n$ constant matrices, $0 \leq m \leq 2 n$, such that the $m$ boundary forms (1.1b) are linearly independent. A necessary and sufficient condition for the latter assumption is that the rows of the $m \times 3 n$ matrix [MNF(x)] are linearly independent on $a \leq x \leq b$ (Jones, [2, Theorem 2.1]).

Now, by a linear rearrangement of its rows, we may place the boundary conditions (1.1b) in the form

$$
\begin{aligned}
M_{0} y(a)+N_{0} y(b) & =0, \\
M_{1} y(a)+N_{1} y(b)+\int_{a}^{b} F_{1}(t) y d t & =0, \\
\int_{a}^{b} F_{2}(t) y d t & =0,
\end{aligned}
$$

where $M_{0}$ and $N_{0}$ are each $\rho \times n$ constant matrices with $\left[M_{0} N_{0}\right]$ having rank $\rho$, $M_{1}$ and $N_{1}$ each $\sigma \times n$ matrices with $\left[M_{1} N_{1}\right]$ having rank $\sigma, F_{1}(x)$ and $F_{2}(x)$, respectively, $\sigma \times n$ and $\tau \times n$ matrices such that the $\sigma+\tau$ rows of $F_{1}(x)$ and $F_{2}(x)$ are a set of $\sigma+\tau$ linearly independent vectors on $[a, b]$, the $\rho+\sigma$ rows of $\left[M_{0} N_{0}\right]$ and $\left[M_{1} N_{1}\right]$ are linearly independent, and $\rho+\sigma+\tau=m$. Moreover, as this transformation may be effected by multiplying (1.1b) on the left by a suitable $m \times m$ nonsingular constant matrix $D$, the partitioning of $K(x) D^{-1} \equiv$ $\left[K_{0}(x) K_{1}(x) K_{2}(x)\right]$ into $\rho, \sigma$ and $\tau$ columns, respectively, in view of the last 
boundary condition of (2.1b), reduces (1.1a) to the form

(2.1a) $A_{1}(x) y^{\prime}+A_{0}(x) y+H(x)\left[M_{2} y(a)+N_{2} y(b)\right]+K_{1}(x) \int_{a}^{b} F_{1}(t) y d t=\lambda B(x) y$.

Furthermore, without loss of generality, we may assume that $p=2 n-(\rho+\sigma)$ and that the $2 n \times 2 n$ matrix

$$
\left[\begin{array}{ll}
M_{0} & N_{0} \\
M_{1} & N_{1} \\
M_{2} & N_{2}
\end{array}\right]
$$

is nonsingular (see, for example, Remark 6.2 of [5]).

Transforming the integro-differential-boundary problem (2.1a), (2.1b) into an equivalent two-point boundary problem in a manner analogous to that employed in $[2],[3]$ and $[5]$ yields the adjoint problem. Introducing new vectors

$$
\begin{array}{ll}
u_{1} \equiv M_{1} y(a)+\int_{a}^{x} F_{1}(t) y d t, & u_{2} \equiv \int_{a}^{x} F_{2}(t) y d t, \\
s_{1} \equiv u_{1}(b)-u_{1}(a)=\int_{a}^{b} F_{1}(t) y d t, & s_{2} \equiv M_{2} y(a)+N_{2} y(b),
\end{array}
$$

problem (2.1a), (2.1b) is equivalent to the system consisting of $(3 n+\sigma+\tau-\rho)$ linear differential equations and $2 n+2(\sigma+\tau)$ end-point conditions:

$$
\begin{gathered}
A_{1}(x) y^{\prime}+A_{0}(x) y+K_{1}(x) s_{1}+H(x) s_{2}=\lambda B(x) y, \\
u_{1}^{\prime}-F_{1}(x) y=0, \quad u_{2}^{\prime}-F_{2}(x) y=0, \\
s_{1}^{\prime}=0, \quad s_{2}^{\prime}=0, \\
M_{0} y(a)+N_{0} y(b)=0, \quad M_{1} y(a)-u_{1}(a)=0, \quad N_{1} y(b)+u_{1}(b)=0, \\
u_{2}(a)=0, \quad u_{2}(b)=0, \\
u_{1}(a)+s_{1}(a)-u_{1}(b)=0, \quad M_{2} y(a)-s_{2}(a)+N_{2} y(b)=0 .
\end{gathered}
$$

Now, if the inverse of (2.2) is introduced and partitioned,

$$
\left[\begin{array}{ll}
M_{0} & N_{0} \\
M_{1} & N_{1} \\
M_{2} & N_{2}
\end{array}\right]\left[\begin{array}{rrr}
-P_{0} & -P_{1} & -P_{2} \\
Q_{0} & Q_{1} & Q_{2}
\end{array}\right]=I_{2 n},
$$


where $P_{0}$ and $Q_{0}$ are each of dimension $n \times \rho, P_{1}$ and $Q_{1}$ each $n \times \sigma$ and $P_{2}$ and $Q_{2}$ each $n \times p$, then the system adjoint to (2.3) is readily obtained (see, for example, $[4, \$ 3.6])$, being comprised of $3 n+\sigma+r-\rho$ differential equations and $4 n-2 \rho$ end-point conditions:

$$
\begin{gathered}
-\left[A_{1}^{*}(x) z\right]^{\prime}+A_{0}^{*}(x) z-F_{1}^{*}(x) v_{1}-F_{2}^{*}(x) v_{2}=\lambda B^{*}(x) z, \\
v_{1}^{\prime}=0, \quad v_{2}^{\prime}=0, \\
-t_{1}^{\prime}+K_{1}^{*}(x) z=0, \quad-t_{2}^{\prime}+H^{*}(x) z=0, \\
P_{2}^{*} A_{1}^{*}(a) z(a)+Q_{2}^{*} A_{1}^{*}(b) z(b)+P_{2}^{*} M_{1}^{*} v_{1}(a)-Q_{2}^{*} N_{1}^{*} v_{1}(b)-t_{2}(a)=0, \\
P_{1}^{*} A_{1}^{*}(a) z(a)+Q_{1}^{*} A_{1}^{*}(b) z(b)+P_{1}^{*} M_{1}^{*} v_{1}(a)-Q_{1}^{*} N_{1}^{*} v_{1}(b)+t_{1}(a)=0, \\
t_{1}(b)=0, \quad t_{2}(b)=0 .
\end{gathered}
$$

Then, eliminating $v_{1}, t_{1}$ and $t_{2}$ with the aid of relation (2.4), the problem

$$
-\left[A_{1}^{*}(x) z\right]^{\prime}+A_{0}^{*}(x) z-F_{1}^{*}(x)\left[P_{1}^{*} A_{1}^{*}(a) z(a)+Q_{1}^{*} \cdot 4_{1}^{*}(b) ;(b)\right]
$$

$$
+F_{1}^{*}(x) \int_{a}^{b} K_{1}^{*}(t) z d t-F_{2}^{*}(x) v_{2}=\lambda B^{*}(x) z, \quad v_{2}=\text { constant }
$$

$$
P_{2}^{*} A_{1}^{*}(a) z(a)+Q_{2}^{*} A_{1}^{*}(b) z(b)+\int_{a}^{b} H^{*}(t) z d t=0
$$

will be defined as the integro-differential-boundary problem adjoint to problem (2.1a), (2.1b).

It is to be noted that, in general, the parameter $v_{2}$ cannot be eliminated from (2.6a). However, for the problems discussed by Krall [3] it was assumed that $r=0$, and, hence, an adjoint problem not involving a parameter may be introduced. In addition, it is readily verifiable that the adjoint problem $(2.6)$ is independent of a reformulation of system (2.1a), (2.1b) into an equivalent problem by the addition to (2.1a) of terms of the form

$$
\begin{gathered}
J_{0}(x)\left[M_{0} y(a)+N_{0} y(b)\right] \\
+J_{1}(x)\left[M_{1} y(a)+N_{1} y(b)+\int_{a}^{b} F_{1}(t) y d t\right]+J_{2}(x) \int_{a}^{b} F_{2}(t) y d t,
\end{gathered}
$$

$J_{i}(x)(i=1,2,3)$ continuous on $[a, b]$. This invariance property of the adjoint will be applied in developing canonical forms in the next section.

A problem (2.1a), (2.1b) will be termed symmetric if the integral-boundary 
forms (2.1b) and (2.6b) are equivalent and the integro-differential-boundary operators in (2.1a) and (2.6a) coincide when applied to vectors of class $C^{\prime}$ satisfying the integro-boundary conditions (2.1b). This terminology is an extension of that applied to differential expressions in $[4, \mathrm{p} .122]$ and corresponds to the definition of selfadjointness in [4, p. 197]. Focusing our attention first on the integralboundary conditions, we have the following generalization of results contained in Theorem 5.1 of [3].

Lemma 2.1. For a problem (2.1a), (2.1b) the integro-boundary forms (2.1b) and (2.6b) of the adjoint problem are equivalent if and only if

(a) $\rho+\sigma=n$ and $\tau=0$, or equivalently, $m=p=n$,

(b) $\left[M_{0}, N_{0} ; M_{1}, N_{1}\right] \cdot \operatorname{diag}\left\{-A_{1}^{*-1}(a), A_{1}^{*-1}(b) \cdot\left[M_{0}, N_{0} ; M_{1}, N_{1}\right]^{*}=0\right.$, and

(c) $H(x) \equiv F_{1}^{*}(x) E_{1}$ on $[a, b]$, where $E_{1} \equiv\left[0 I_{\sigma}\right] E, E \equiv C^{-1}$, and

$$
C=\left[M_{2} N_{2}\right] \cdot \operatorname{diag}\left\{-A_{1}^{-1}(a), A_{1}^{-1}(b)\right\} \cdot\left[M_{0}, N_{0} ; M_{1}, N_{1}\right]^{*} \text {. }
$$

As the rank of the $p \times 2 n$ matrix $\left[P_{2}^{*} A_{1}^{*}(a) Q_{2}^{*} A_{1}^{*}(b)\right]$ is $p$, equivalence of the integro-boundary forms (2.1b) and (2.6b) requires that $m=p=\rho+\sigma$, whence (2.7a) follows. Then, under (2.7a) a necessary and sufficient condition for equivalence of the integro-boundary forms is the existence of an $n \times n$ constant nonsingular matrix $E$ such that

$$
\begin{gathered}
{\left[P_{2}^{*} A_{1}^{*}(a) Q_{2}^{*} A_{1}^{*}(b)\right]=E^{*}\left[M_{0}, N_{0} ; M_{1}, N_{1}\right]} \\
H^{*}(x) \equiv E^{*}\left[0 F_{1}^{*}(x)\right]^{*} \text { on }[a, b] .
\end{gathered}
$$

Now, it follows from relation (2.4) that (2.7b) and (2.8) are equivalent with $E$ as given in (2.7c). For this latter equivalence we first note that, as (2.7b) is an immediate consequence of (2.8), the nonsingularity of the matrix $C$, defined in (2.7c), follows either by a method analogous to that used in [3, p. 445] or, as (2.7b) is an immediate consequence of (2.8) for some nonsingular $E$, from the fact that the rows of $\left[M_{2} N_{2}\right]$ are linearly independent of the rows of $\left[M_{0}, N_{0} ; M_{1}, N_{1}\right]$, while the latter rows constitute a maximal linearly independent vector set orthogonal to the $2 n$ columns of diag $\left.-A_{1}^{-1}(a), A_{1}^{-1}(b)\right\} \cdot\left[M_{0}, N_{0} ; M_{1}, N_{1}\right]^{*}$. Then, with $E$ defined as in (2.7c), (2.8) follows from (2.7b) and the fact that the matrices $P_{2}$ and $Q_{2}$ are uniquely determined by the relations (2.4). Finally, (2.7c) and (2.9) are clearly equivalent under the definition of $E_{1}$ in (2.7c). 
Lemma 2.2. Under equivalence of the integro-boundary forms (2.1b) and (2.6b), problem (2.1a), (2.1b) is symmetric if and only if there exists $a \sigma \times \sigma$ constant Hermitian matrix $\Gamma$ such that on $a \leq x \leq b$
(a) $A_{1}^{*}(x) \equiv-A_{1}(x), A_{0}^{*}(x) \equiv A_{0}(x)-A_{1}^{\prime}(x), B^{*}(x) \equiv B(x)$,
(b) $K_{1}(x) \equiv F_{1}^{*}(x)[\Gamma+1 / 2 \Theta], \Theta \equiv P_{1}^{*} A_{1}(a) P_{1}-Q_{1}^{*} A_{1}(b) Q_{1}$.

For a symmetric problem the relations in the first condition follow from termby-term identification of corresponding operators when applied to vectors $y$ of class $C^{\prime}$ satisfying $y(a)=y(b)=\int_{a}^{b} F_{1}(t) y d t=\int_{a}^{b} K_{1}^{*}(t) y d t=0$. Then, under (2.7) and (2.10a), a necessary and sufficient condition for problem (2.1a), (2.1b) to be symmetric is that

$$
\begin{aligned}
F_{1}^{*}(x)\left[\left(E_{1} M_{2}+\right.\right. & \left.\left.P_{1}^{*} A_{1}^{*}(a)\right) y(a)+\left(E_{1} N_{2}+Q_{1}^{*} A_{1}^{*}(b)\right) y(b)\right] \\
& -F_{1}^{*}(x) \int_{a}^{b} K_{1}^{*}(t) y d t+K_{1}(x) \int_{a}^{b} F_{1}(t) y d t \equiv 0
\end{aligned}
$$

on $[a, b]$ for arbitrary vectors $y$ of class $C^{\prime}$ satisfying (2.1b), wherein $\tau=0$. Now, for vectors $y \in C^{\prime}$ such that $y(a)=y(b)=\int_{a}^{b} F_{1}(t) y d t=0$, we have that $F_{1}^{*}(x) \int_{a}^{b} K_{1}^{*}(t) y d t=0$; and, as the $\sigma$ columns of $F_{1}^{*}(x)$ are linearly independent on $[a, b], \int_{a}^{b} K_{1}^{*}(t) y d t=0$. Consequently, it follows that $\int_{a}^{b} K_{1}^{*}(t) v d t=0$ for arbitrary continuous vectors $v(t)$ satisfying $\int_{a}^{b} F_{1}(t) v d t=0$. In addition, as $\int_{a}^{b} F_{1}(t) F_{1}^{*}(t) d t$ is nonsingular, let the $\sigma \times \sigma$ constant matrix $\Phi$ be determined by $\int_{a}^{b} F_{1}(t)\left[K_{1}(t)-F_{1}^{*}(t) \Phi\right] d t=0$. Then, for $v(t) \equiv\left[K_{1}(t)-F_{1}^{*}(t) \Phi\right] c, c$ an arbitrary $\sigma$-dimensional constant vector, we have that $\int_{a}^{b}\left[K_{1}^{*}(t)-\Phi^{*} F_{1}(t)\right] v(t) d t=0$, and, consequently,

$$
K_{1}(x) \equiv F_{1}^{*}(x) \Phi \quad \text { on }[a, b]
$$

Moreover, under (2.12), in view of the linear independence of the columns of $F_{1}^{*}(x)$ on $[a, b]$, condition (2.11) for vectors $y \in C^{\prime}$ satisfying (2.1b) with $r=0$ reduces to the requirement that

$$
\left(E_{1} M_{2}+P_{1}^{*} A_{1}^{*}(a)-\Theta M_{1}\right) y(a)+\left(E_{1} N_{2}+Q_{1}^{*} A_{1}^{*}(b)-\Theta N_{1}\right) y(b)=0
$$

where $\Theta \equiv \Phi-\Phi^{*}$, for arbitrary vectors $y(a), y(b)$ such that $M_{0} y(a)+N_{0} y(b)=0$. Now, as the $n+\sigma$ columns of $\left[-P_{1},-P_{2} ; Q_{1}, Q_{2}\right]$ form a maximal set of linearly independent vectors orthogonal to the $\rho$ rows of $\left[M_{0} N_{0}\right]$, relation (2.13) for $y(a)$, $y(b)$ satisfying $M_{0} y(a)+N_{0} y(b)=0$ holds if and only if 


$$
\begin{aligned}
& -\left(E_{1} M_{2}+P_{1}^{*} A_{1}^{*}(a)-\Theta M_{1}\right) P_{1}+\left(E_{1} N_{2}+Q_{1}^{*} A_{1}^{*}(b)-\Theta N_{1}\right) Q_{1}=n \\
& -\left(E_{1} M_{2}+P_{1}^{*} A_{1}^{*}(a)-\Theta M_{1}\right) P_{2}+\left(E_{1} N_{2}+Q_{1}^{*} A_{1}^{*}(b)-\Theta N_{1}\right) Q_{2}=0 ;
\end{aligned}
$$

or, equivalently, if and only if

$$
\begin{aligned}
& \Theta=-P_{1}^{*} A_{1}^{*}(a) P_{1}+Q_{1}^{*} A_{1}^{*}(b) Q_{1}, \\
& E_{1}=P_{1}^{*} A_{1}^{*}(a) P_{2}-Q_{1}^{*} A_{1}^{*}(b) Q_{2} .
\end{aligned}
$$

However, (2.16) reduces to an identity under (2.8) and (2.10a). Consequently, on setting $\Gamma \equiv 1 / 2\left(\Phi+\Phi^{*}\right)$ we have $\Phi=\Gamma+1 / 2 \Theta$, and the lemma is established.

Moreover, as conditions (2.14) are equivalent to the existence of an $\sigma \times \rho$ constant matrix $J$ such that

$$
E_{1} M_{2}+P_{1}^{*} A_{1}^{*}(a)-\Theta M_{1}=J M_{0}, \quad E_{1} N_{2}+Q_{1}^{*} A_{1}^{*}(b)-\Theta N_{1}=J N_{0},
$$

on substituting the values of $P_{1}$ and $Q_{1}$ from (2.17) into (2.15) it follows, from the definition of $E_{1}$ in $(2.7 \mathrm{c})$, that

$$
\Theta=E_{1}\left(-M_{2} A_{1}^{*-1}(a) M_{2}^{*}+N_{2} A_{1}^{*-1}(b) N_{2}^{*}\right) E_{1}^{*}
$$

On the other hand, for $K_{1}(x)$ of the form (2.12) with $\Phi=\Gamma+1 / 2 \Theta, \Gamma$ a $\sigma \times \sigma$ constant Hermitian matrix and $\Theta$ given by (2.18), we have from (2.4) and (2.7b) that $P_{1}$ and $Q_{1}$ satisfy relations (2.17) with $J \equiv E_{1}\left(-M_{2} A_{1}^{*-1}(a) M_{2}^{*}+\right.$ $\left.N_{2} A_{1}^{*-1}(b) N_{2}^{*}\right) E_{0}^{*}, E_{0} \equiv\left[I_{\rho} 0\right] E$, and that $\Theta$ satisfies (2.15). Combining these facts with the two lemmas yields the following result.

Theorem 2.1. The integro-differential-boundary problem (2.1a), (2.1b) is symmetric (selfadjoint) if and only if there exists a $\sigma \times \sigma$ constant Hermitian matrix $\Gamma$ sucb that on $a \leq x \leq b$
(a) $\rho+\sigma=n$ and $r=0$,
(b) $\left[M_{0}, N_{0} ; M_{1}, N_{1}\right] \cdot \operatorname{diag}\left\{-A_{1}^{-1}(a), A_{1}^{-1}(b)\right\} \cdot\left[M_{0}, N_{0} ; M_{1}, N_{1}\right]^{*}=0$,
(c) $A_{1}^{*}(x) \equiv-A_{1}(x), A_{0}^{*}(x) \equiv A_{0}(x)-A_{1}^{\prime}(x), B^{*}(x) \equiv B(x)$,
(d) $H(x) \equiv F_{1}^{*}(x) E_{1}$,
(e) $K_{1}(x) \equiv F_{1}^{*}(x)[\Gamma+1 / 2 \Theta]$, 
where $E_{1}$ is given in (2.7c) and $\Theta$ in (2.18).

For the special case $K_{1}(x) \equiv 0$ considered by Krall [3], condition (2.19e) reduces to $\Theta=0$ in view of the linear independence of the columns of $F_{1}^{*}(x)$ on $[a, b]$. This simplification of the condition corresponding to condition (5.7) of Theorem 5.1 of [3] accrues from the above formulation of the integro-boundary conditions notwithstanding the more restrictive equivalence of terms under selfadjointness in [3].

Corollary. An integro-differential-boundary problem (2.1a), (2.1b) with $K_{1}(x) \equiv 0$ on $[a, b]$ is symmetric if and only if relations $(2.19 a, b, c, d)$ bold on $[a, b]$ and $E_{1}\left(M_{2} A_{1}^{-1}(a) M_{2}^{*}-N_{2} A^{-1}(b) N_{2}^{*}\right) E_{1}^{*}=0, E_{1}$ defined in (2.7c).

3. Canonical forms. For a symmetric integro-differential-boundary problem

$$
\begin{gathered}
A_{1}(x) y^{\prime}+A_{0}(x) y+H(x)\left[M_{2} y(a)+N_{2} y(b)\right]+K_{1}(x) \int_{a}^{b} F_{1}(t) y d t=\lambda B(x) y, \\
M_{0} y(a)+N_{0} y(b) \quad=0, \\
M_{1} y(a)+N_{1} y(b)+\int_{a}^{b} F_{1}(t) y d t=0,
\end{gathered}
$$

with the $\rho \times 2 n$ matrix $\left[M_{0} N_{0}\right]$, the $\sigma \times 2 n$ matrix $\left[M_{1} N_{1}\right]$, the $n \times 2 n$ matrix $\left[M_{2} N_{2}\right]$ and the $2 n \times 2 n$ matrix (2.2) each having maximal rank, and the $\sigma$ rows of $F_{1}(x)$ linearly independent on $[a, b]$, it may be assumed, without loss of generality, that the orthogonality condition

$$
M_{0} M_{1}^{*}+N_{0} N_{1}^{*}=0
$$

holds. For, as the $\rho \times \rho$ matrix $W_{0} \equiv M_{0} M_{0}^{*}+N_{0} N_{0}^{*}$ is nonsingular, the orthogonality relation (3.2) prevails upon adding to the last $\sigma$ integro-boundary conditions of (3.1) the first $\rho$ conditions premultiplied by $-\left(M_{1} M_{0}^{*}+N_{1} N_{0}^{*}\right) W_{0}^{-1}$; or equivalently, by premultiplying the integro-boundary conditions of (3.1) by the $n \times n$ nonsingular matrix

$$
\left[I_{\rho}, 0 ;-\left(M_{1} M_{0}^{*}+N_{1} N_{0}^{*}\right) W_{0}^{-1}, I_{\sigma}\right]
$$

Moreover, inasmuch as the new integro-boundary conditions are equivalent to the original and the matrices $H(x), K_{1}(x), F_{1}(x), M_{0}, N_{0}, M_{2}, N_{2}, P_{1}, Q_{1}, P_{2}, Q_{2}$, $E_{1}$ and $\Theta$ are unaffected by this change of $M_{1}$ and $N_{1}$, conditions (2.19) remain valid and the symmetry of the problem is preserved. It is to be noted that while the matrix $C$ undergoes a postmultiplication by the conjugate-transpose of (3.3) 
the matrix $E_{1}$ remains unaltered. In this section we sball assume that the ortbogonality condition (3.2) bolds.

Now, as

$$
-E_{1} M_{2} A_{1}^{-1}(a) M_{0}^{*}+E_{1} N_{2} A_{1}^{-1}(b) N_{0}^{*}=0
$$

from the definition of $E_{1}$ in (2.7c), and a maximal set of $2 n-\rho$ linearly independent vectors of the null space of $\left[-M_{0} A_{1}^{*-1}(a) N_{0} A_{1}^{*-1}(b)\right]$ is given by the columns of the $2 n \times(n+\sigma)$ matrix

$$
\left[\begin{array}{rrr}
M_{0}^{*} & M_{1}^{*} & -A_{1}^{*}(a) M_{1}^{*} \\
N_{0}^{*} & N_{1}^{*} & A_{1}^{*}(b) N_{1}^{*}
\end{array}\right]
$$

in view of (2.19b) and (3.2), it follows that there exist $\sigma \times \rho, \sigma \times \sigma$ and $\sigma \times \sigma$ constant matrices $R, S$ and $T$, respectively, such that

$$
E_{1} M_{2}=R M_{0}+S M_{1}-T M_{1} A_{1}(a), \quad E_{1} N_{2}=R N_{0}+S N_{1}+T N_{1} A_{1}(b)
$$

On postmultiplying these two relations by $-A_{1}^{-1}(a) M_{1}^{*}$ and $A_{1}^{-1}(b) N_{1}^{*}$, respectively, and adding, we have that $I_{\sigma}=T W_{1}$, where

$$
W_{1} \equiv M_{1} M_{1}^{*}+N_{1} N_{1}^{*}
$$

is a $\sigma \times \sigma$ nonsingular Hermitian matrix; and, hence, $T=W_{1}^{-1}$. Then, on postmultiplying relations (3.4), in turn, by $M_{1}^{*}, N_{1}^{*}$ and $-A_{1}^{-1}(a) M_{2}^{*} E_{1}^{*}, A_{1}^{-1}(b) N_{2}^{*} E_{1}^{*}$, respectively, and adding, it follows from (2.18), (2.19b), (3.2) and the definition of $E_{1}$ that the matrix $S$ has the representations:

$$
S=\Omega+E_{1}\left(M_{2} M_{1}^{*}+N_{2} N_{1}^{*}\right) W_{1}^{-1}, \quad S=\Theta+W_{1}^{-1}\left(M_{1} M_{2}^{*}+N_{1} N_{2}^{*}\right) E_{1}^{*},
$$

where

$$
\Omega \equiv W_{1}^{-1}\left(M_{1} A_{1}(a) M_{1}^{*}-N_{1} A_{1}(b) N_{1}^{*}\right) W_{1}^{-1}
$$

As $S-S^{*}=\Theta+\Omega$, the matrix $S-1 / 2 \Theta-1 / 2 \Omega$ is Hermitian; and, consequently, 


$$
\begin{aligned}
& H(x)\left[M_{2} y(a)+N_{2} y(b)\right]+K_{1}(x) \int_{a}^{b} F_{1}(t) y d t \\
& =F_{1}^{*}(x)\left\{W_{1}^{-1}\left[-M_{1} A_{1}(a) y(a)+N_{1} A_{1}(b) y(b)\right]+1 / 2 \Omega\left[M_{1} y(a)+N_{1} y(b)\right]\right\} \\
& \quad+F_{1}^{*}(x)[\Gamma+1 / 2 \Theta+1 / 2 \Omega-S] \int_{a}^{b} F_{1}(t) y d t
\end{aligned}
$$

for vectors $y \in C$ satisfying the integro-boundary conditions of (3.1). The invariance property of the adjoint problem, discussed in the previous section, then assures the following result.

Theorem 3.1. Every symmetric integrodifferential-boundary problem (3.1) is representable in the form

$A_{1}(x) y^{\prime}+A_{0}(x) y+F_{1}^{*}(x)\left\{w_{1}^{-1}\left[-M_{1} A_{1}(a) y(a)+N_{1} A_{1}(b) y(b)\right]+1 / 2 \Omega\left[M_{1} y(a)+N_{1} y(b)\right]\right\}$

$$
+F_{1}^{*}(x) \Psi \int_{a}^{b} F_{1}(t) y d t=\lambda B(x) y,
$$

$$
\begin{array}{ll}
M_{0} y(a)+N_{0} y(b) & =0, \\
M_{1} y(a)+N_{1} y(b)+\int_{a}^{b} F_{1}(t) y d t=0,
\end{array}
$$

where $\Psi$ is a $\sigma \times \sigma$ constant Hermitian matrix, $w_{1}$ is defined by (3.5) and $\Omega$ by (3.6), the orthogonality relations (2.19b) and (3.2) bold, and the $\sigma$ rows of $F_{1}(x)$ are linearly independent and the matrices $A_{1}(x), A_{0}(x)$ and $B(x)$ satisfy (2.19c) on $[a, b]$.

Conversely, a problem (3.7) with the $\rho$ rows of $\left[M_{0} N_{0}\right]$ and the $\sigma$ rows of $\left[M_{1} N_{1}\right]$ constituting $\rho+\sigma=n$ linearly independent rows satisfying $(2.19 \mathrm{~b})$ and (3.2), $\Psi$ a $\sigma \times \sigma$ constant Hermitian matrix, $W_{1}$ and $\Omega$ given by (3.5) and (3.6), respectively, and for which relations $(2.19 c)$ hold and the $\sigma$ rows of $F_{1}(x)$ are linearly independent on $[a, b]$, is symmetric and coincides with its adjoint problem term-by-term.

Moreover, for a symmetric problem (3.7) satisfying the conditions listed in Theorem 3.1 the rows of the end-point coefficient matrix $\left[M_{0}, N_{0} ; M_{1}, N_{1}\right]$ may be orthonormalized, in view of condition (3.2), by premultiplying the two sets of integro-boundary conditions by the unique positive square roots of $w_{0}^{-1}$ and $w_{1}^{-1}$, respectively. The resulting system may then be said to be in canonical form.

Corollary. Every symmetric integro-differential-boundary problem (3.1) is reducible to the form 


$$
\begin{aligned}
A_{1}(x) y^{\prime}+ & A_{0}(x) y+F_{1}^{*}(x)\left[-M_{1} A_{1}(a) y(a)+N_{1} A_{1}(b) y(b)\right] \\
+ & F_{1}^{*}(x)[\Psi+\Lambda] \int_{a}^{b} F_{1}(t) y d t=\lambda B(x) y, \\
M_{0} y(a)+N_{0} y(b) \quad & 0, \\
M_{1} y(a)+N_{1} y(b)+\int_{a}^{b} F_{1}(t) y d t & =0
\end{aligned}
$$

where $\Lambda \equiv(1 / 2)\left(-M_{1} A_{1}(a) M_{1}^{*}+N_{1} A_{1}(b) N_{1}^{*}\right), \Psi$ is a $\sigma \times \sigma$ constant Hermitian matrix, relations (2.19c) bold and the $\sigma$ rows of $F_{1}(x)$ are linearly independent on $[a, b]$, the rows of the $n \times 2 n$ matrix $\left[M_{0}, N_{0} ; M_{1}, N_{1}\right]$ are ortbonormalized in the sense that $M_{0} M_{0}^{*}+N_{0} N_{0}^{*}=I_{\rho}, M_{1} M_{1}^{*}+N_{1} N_{1}^{*}=I_{\sigma}$ and $M_{0} M_{1}^{*}+N_{0} N_{1}^{*}=0$, and

$$
\left[M_{0}, N_{0} ; M_{1}, N_{1}\right] \cdot \operatorname{diag}\left\{-A^{-1}(a), A^{-1}(b)\right\} \cdot\left[M_{0}, N_{0} ; M_{1}, N_{1}\right]^{*}=0
$$

In particular, if $\Lambda=0$ for a symmetric problem (3.1), as it does, for example, if $A_{1}(x)$ is a constant multiple of a unitary matrix, the forms (3.7) and (3.8) are further reduced.

\section{REFERENCES}

1. R. H. Cole, General boundary conditions for an ordinary differential system, Trans. Amer. Math. Soc. 111 (1964), 521-550. MR 28 \#3193.

2. W. R. Jones, Differential systems with integral boundary conditions, J. Differential Equations 3 (1967), 191-202. MR 34 \#6207.

3. A. M. Krall, Differential-boundary operators, Trans. Amer. Math. Soc. 154 (1971), 429-458. MR 42 \#6328.

4. W. T. Reid, Ordinary differential equations, Wiley, New York, 1971. MR 42 $\# 7963$.

5. O. Vejvoda and M. Tvrdý, Existence of solutions to a linear integro-boundarydifferential equation with additional conditions, Ann. Mato Pura Applo set. (4), 89 (1971), 169-216.

DEPARTMENT OF MATHEMATICS, HILL CENTER FOR THE MATHEMATICAL SCIENCES, RUTGERS UNIVERSITY, NEW BRUNSWICK, NEW JERSEY 08903 